\title{
Suplementação de oxigênio e prevenção na retinopatia da prematuridade
}

\author{
Oxygen supplementation and prevention in retinopathy of prematurity
}

Administración de suplementos de oxígeno y prevención en retinopatía del prematuro

Taíssa Naves Araújo ${ }^{1 *}$, Juarez Naves Araújo², Milena Bentivoglio Cunha Naves Vasconcelos ${ }^{3}$, Ruy Rodrigues Naves Martins Soares ${ }^{4}$, Lindomar Guedes Freire Filha ${ }^{5}$.

\section{RESUMO}

Objetivo: Realizar uma revisão literária sobre os métodos de investigação, o rastreio e a prevenção da Retinopatia da Prematuridade (ROP) desencadeada pela suplementação de oxigênio excessivo. Revisão Bibliográfica: A visão da espécie humana é relevante e para a criança tem papel ímpar na evolução físicacognitiva normal. Nos prematuros observou-se que a ROP muitas vezes pode ser subdiagnosticada e subestimada. Devem ser implementados e seguidos protocolos de rastreio de danos da prematuridade em todas as Unidades Neonatais. Além disso, treinar a equipe multidisciplinar a fim de tentar minimizar os riscos associados à suplementação de oxigênio, principalmente em prematuros. Existe um importante projeto, Controlando Oxigênio Alvo Ativamente (Projeto COALA), o qual tenta evitar suplementações excessivas de oxigênio em recém-nascidos. Considerações finais: É desafiador controlar a quantidade de oxigênio ofertada aos prematuros frente à quantidade de oxigênio necessária em várias outras patologias decorrentes deste período neonatal. Assim, inferiu-se que para uma melhor prevenção de Retinopatia da Prematuridade deve-se ter treinamento e capacitação de uma equipe multidisciplinar em Unidades Neonatais.

Palavras-chave: Retinopatia, Prematuridade, Rastreio, Prevenção.

\begin{abstract}
Objective: To accomplish a literature review on investigation methods, screening and prevention of Retinopathy of Prematurity (ROP) triggered by excessive oxygen supplementation. Bibliographic Review: The view of the human species is relevant and for the child it has a unique role in normal physical-cognitive evolution. In preterm infants, it was observed that ROP can often be underdiagnosed and underestimated. It was emphasized that prematurity damage screening protocols must be implemented and followed in all Neonatal Units. In addition, a multidisciplinary team must be trained in order to try to minimize the risks associated with oxygen supplementation, especially in preterm infants. There is an important project, Actively Controlling Target Oxygen (Project COALA), which tries to prevent excessive oxygen supplementation in newborns Final considerations: It is challenging to control the amount of oxygen offered to preterm infants compared to the amount of oxygen needed in several other pathologies resulting from this neonatal period. Thus, it was inferred that for a better prevention of Retinopathy of Prematurity, training and qualification of a multidisciplinary team in Neonatal Units must be carried out.
\end{abstract}

Keywords: Retinopathy, Premature, Screening, Prevention.

\footnotetext{
${ }_{1}$ Santa Casa de Misericórdia de Franca, Franca - SP. *E-mail: taissanaves@hotmail.com

2 Unidade Paulista de Oftalmologia, São Paulo - SP

3 Universidade Nove de Julho (UNINOVE), Guarulhos - SP.

${ }^{4}$ Faculdade Ceres (FACERES), São José do Rio Preto - SP.

${ }^{5}$ Faculdade Delta, Goiânia - GO.
} 


\section{RESUMEN}

Objetivo: Lograr una revisión literaria sobre métodos de investigación, el cribado y prevención de la Retinopatía del Prematuro (ROP) motivado por suplementación excesiva de oxígeno. Revisión bibliográfica: La visión en la especie humana es relevante y para el niño tiene un papel único en la evolución físico-cognitiva normal. En los bebés prematuros, se observó que la ROP a menudo se puede infradiagnosticar y subestimar. Se enfatizó que se deben implementar y seguir protocolos de detección de daños en la prematuridad en todas las Unidades Neonatales. Además, se debe capacitar a un equipo multidisciplinario para tratar de minimizar los riesgos asociados con la suplementación con oxígeno, especialmente en bebés prematuros. Existe un proyecto importante, Control Activo de Oxígeno Objetivo (Proyecto COALA), que intenta prevenir la suplementación excesiva de oxígeno en los recién nacidos. Consideraciones finales: Es un desafío controlar la cantidad de oxígeno que se ofrece a los bebés prematuros en comparación con la cantidad de oxígeno necesaria en varias otras patologías que resultan de este período neonatal. Así, se infirió que para una mejor prevención de la retinopatía de prematuros.

Palabras clave: Retinopatía, Prematuro, Seguimiento, Prevención.

\section{INTRODUÇÃO}

A retinopatia da prematuridade (ROP) é uma doença ocular desenvolvida na retina dos recém-nascidos (RNs), principalmente os pré-termos, causada por multiplicação anormal de vasos na área. Esta patologia é considerada entre as maiores causas de cegueira infantil (CARRION JL, et al., 2011).

Criança com boa acuidade visual apresenta desenvolvimento físico e cognitivo melhores, visto que a visão participa do desenvolvimento de gestos e condutas sociais, observa os movimentos, entende ações, interage e aprende a manejar objetos ao longo de sua evolução. A interpretação da imagem, é através da captação dos estímulos luminosos dos olhos, a qual é enviada para o córtex cerebral para interpretação e formação da imagem. Sendo assim, um obstáculo à formação de imagens nítidas acarretará em uma deficiência no desenvolvimento visual, o qual pode tornar-se inconvertível se não tratado em tempo hábil (WEBER $\mathrm{J} e$ KELLEY J, 2014; FRANÇA-FREITAS MP e GIL MSCA, 2012).

Crianças portadores de Retinopatia da Prematuridade (ROP) apresentam maior probabilidade de desenvolver déficits cognitivos e motores abaixo da média esperada aos 2 anos de idade. Essa visão é deficitária ao nascimento, visto a imaturidade do cérebro e da retina. A visão monocular define-se ao final dos primeiros 60 dias de vida e a visão binocular se estabelece totalmente ao fim dos seis a oito primeiros meses de vida (SOCIEDADE DE PEDIATRIA DE SÃO PAULO, 2017).

A ROP apresenta causas multifatoriais, as quais interferem na vascularização retiniana da periferia. Em geral os principais fatores de risco são: prematuridade, baixo peso ao nascer e suplementação de oxigênio excessiva, dentre outros (FONTES FILHO JB, et al., 2011).

Alguns trabalhos iniciais foram realizados na Austrália por Campbell no ano de 1951 e na Inglaterra por Crosse no ano de 1952, que correlacionaram a doença com o oxigênio $\left(\mathrm{O}_{2}\right)$, fato histórico que direcionou modificações às condutas clínicas da ROP. Ainda atualmente essa retinopatia continua sendo importante causa de cegueira infantil principalmente na América Latina. Desde então, observa-se que a maioria das pesquisas concentrou na investigação de padrão de vascularização retiniana anormais e incluíram recentemente estudos que corroboram com o aparecimento de ROP, na mudança da arquitetura celular da retina $O$ e vias centrais relevantes, incluindo o córtex visual primário e áreas corticais visuais extra-estriadas (SPANDAU U e KIM SJ, 2019; TARTARELLA MB e FONTES FO JB, 2016).

Ocorreu na história, três grandes períodos de epidemia de ROP. A primeira epidemia, nos anos de 1940, ocorreu em vigência de administração de oxigênio liberal. A segunda epidemia, nos anos de 1960, a qual foi associada ao aumento de sobrevida de RNs de muito baixo peso. E, por fim, a terceira epidemia, em meados de 1980, decorrente da sobrevida de RNs com maior prematuridade. Contudo ainda há efeitos surtidos da 
terceira epidemia em países subdensenvolvidos. No que tange a esse assunto, um estudo que reuniu 70 pediatras que avaliou o nível de consciência, conhecimento, atitude e prática sobre ROP em 11 hospitais na Cisjordânia (Palestina), revelou entre os participantes que $29(41,4 \%)$ dos pediatras não sabiam quando deveria ser iniciada a triagem da ROP (AKKAWI MT, et al., 2018; GILBERT C, et al., 2005).

Globalmente estima-se que haja 1,5 milhões de crianças cegas, dentre estas, 50.000 de ROP, e na América Latina é expressiva causa de cegueira na infância. Essa patologia está correlacionada com as taxas de nascimento e de sobrevivência de RN pré-termo (RNPT) e da triagem para detecção do distúrbio de visão em questão. No Brasil, houve uma queda na taxa de mortalidade neonatal (TMN) de 25,27/1.000 nascidos vivos (nv) em 1990,5 para 7,85 óbitos/1.000 nv em 2019,5, uma estimativa de acordo com a World Health Organization (WHO) e de partos prematuros de extremo baixo peso (PEBP, peso inferior a $1000 \mathrm{~g}$ ), os quais tem graves consequências médico-social e é grande o desafio para o decréscimo dessa taxa nas regiões brasileiras (WHO, 2019; MINISTÉRIO DA SAÚDE, 2013a; ZIN A e GOLE AG, 2013).

No que tange sobre a sobrevivência de pré-termos de muito baixo peso (PMBP, $\leq 1.500 \mathrm{~g}$ ) e de PEBP, devido as coberturas das políticas e programas instituídos mundialmente (como hospitais habilitados para melhoria na qualidade assistencial), as estimativas demonstram que houve um crescimento da sobrevivência PEBP de 8\% para aproximado 35\% (WHO, 2019).

Em países de economia baixa e média, a carga da doença pode variar amplamente entre si e até no mesmo país. A ROP pode afetar até $34 \%$ dos bebês prematuros com peso inferior a 1.500 gramas ao nascer, dos quais 6 a 27\% precisarão de tratamento. Uma revisão sistemática identificou as prevalências de ROP na América Latina: Argentina (2010): 26,2\% de todos os bebês prematuros; Bolívia (2002): 14,3\%; Brasil (2010) 9,3\%; Chile (2004): 12,3\%; Cuba (2010): 5,1\%; Guatemala (2010): 13\%; Nicarágua (2004): 23,8\%; Peru (2007): 19,1\%. Dados do México (2011) relatam uma prevalência de 9,4\% e da Colômbia (2016) mostram $3,19 \%$ por 10.000 nascidos vivos (nv). Com esses dados, a OMS, por meio de seu programa Visão 2020, inclui a gestão dessa entidade dentro de suas políticas prioritárias para melhorar a saúde visual e reduzir a prevalência de cegueira evitável (ORGANIZACIÓN PANAMERICANA DE LA SALUD (OPAS), 2018).

Embora haja avanços significativos na medicina e tecnologia, a considerável prevalência de nascimentos prematuros e riscos de ROP (consequentemente), também é registrada em países desenvolvidos. Blencowe $\mathrm{H}$, et al. (2016), descrevem que configurações de baixa mortalidade neonatal, como nos Estados Unidos da América e Reino Unido, onde ROP é encontrada predominantemente em prematuros extremos. Isso geralmente é detectado por meio de programas de triagem, mas ocorre alguma deficiência visual apesar do tratamento.

As principais diretrizes e protocolos elaborados por instituições internacionais e nacionais (Academia, Associações, Sociedade, Conselhos de Pediatria ou Oftalmologia Pediátrica) apresentam algumas divergências quanto a Idades Gestacionais (IG) e peso de nascimento (PN) para rastreamento. Porém, a maioria das Unidades Neonatais Brasileiras, segue o que a Sociedade Brasileira de Pediatria propõe: 0 rastreio em IG menor que 32 semanas e/ou PN inferior à 1500 g (MINISTÉRIO DA SAÚDE, 2017; CONSELHO BRASILEIRO DE OFTALMOLOGIA, 2017/2018).

Santos MA (2019) em seu estudo reforça a recomendação que a criança prematura deva ser examinada a cada seis meses, e seguir, anualmente as consultas oftalmológicas. A Sociedade de Pediatria de São Paulo (2017) recomenda que a criança prematura deva ser examinada até completar dois anos, a cada seis meses, e posteriormente, anualmente.

Mudanças ocorreram desde a descrição da ROP, dentre elas incluem-se a fisiopatologia, causa, tratamento e prevenção. É indiscutível a necessidade de tratamento de RNPT com oxigenoterapia, entretanto é desafiadora a implementação da atenção neonatal e os programas de triagem e tratamento, principalmente naquelas crianças que receberam oxigênio, a qual pode contribuir com ocorrência de ROP. Diante dessas abordagens o presente artigo teve por objetivo descrever a investigação, o rastreio e a prevenção da retinopatia da prematuridade devido à suplementação de oxigênio excessiva. 


\section{REVISÃO BIBLIOGRÁFICA}

\section{Fisiopatologia}

Retina é uma das estruturas do olho formada por camada de tecido nervoso que recobre internamente o globo ocular na porção posterior, realizando a captação e formação de imagens. O desenvolvimento de vasos retinianos se inicia após a 16 $6^{\underline{a}}$ semana de gestação. Esse crescimento ocorre do centro (nervo óptico) em direção à periferia da retina. Esta vascularização é alcançada ao redor de $34^{a}-36^{\text {a }}$ semana de gestação,

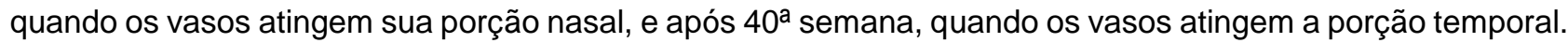
Atinge assim seu desenvolvimento máximo, com etapas essenciais após 25 a 38 semanas pós natal (CERVELLINI PM e SOUZA ABG, 2015).

A ROP é relacionada ao fator de crescimento endotelial vascular regulado por oxigênio e ao fator de crescimento semelhante à insulina-I. Após nascimento prematuro, a suplementação de oxigênio induz a hiperóxia da retina, condição que leva a vasoconstricção e a uma definitiva interrupção da vasculogênese da retina. A isquemia retiniana periférica estimula a neovascularização e surgimento de demais complicações, a qual em determinado momento, pode até resultar num descolamento da retina (SPANDAU U e KIM SJ, 2019).

\section{Rastreio clínico}

É preconizado pela Sociedade Brasileira de Pediatria o teste de triagem oftalmológica inicial: teste do olhinho ou teste do reflexo vermelho (TRV). Este teste é realizado inicialmente por oftalmologistas ou pediatras treinados com ajuda de oftalmocópico direto quando $\mathrm{RN}$ de qualquer idade gestacional nascem. Investiga catarata, glaucoma congênito, tumores intra-oculares, hemorragias intra-vítreas, dentre outros. A técnica para realização pertinente consiste em o examinador encontrar-se em um ambiente escuro e posicionar a transluminação do oftalmoscópio em uma distância de 30 a $40 \mathrm{~cm}$ do RN em ambos os olhos, considerado teste positivo quando os dois olhos apresentam caráter simétrico de luz vermelha (MINISTÉRIO DA SAÚDE, 2013b).

Assegura-se que o TRV faz parte do protocolo instituído pelo Ministério da Saúde e do Sistema Único de Saude (SUS), onde as Diretrizes de Atenção à Saúde Ocular direcionam à Infância. Assim é garantido que todo RN quando nasce, tenha pelo menos um teste do olhinho realizado ainda na Unidade Neonatal ou Maternidade. Para este teste também, os pediatras ou neonatologistas devem ser treinados para realizá-lo (EJZENBAUM J, et al., 2018).

Entretanto, este teste não substitui a oftalmoscopia indireta, quando RN possui os fatores de riscos para rastreio de ROP. Desde 2007, as Diretrizes para a Triagem de Detecção e Tratamento da ROP, indica-se o rastreio em PN menor ou 1500 gramas e/ou IG menor ou igual a 32 semanas; e recomenda-se iniciar com 46 semanas de vida (MINISTÉRIO DA SAÚDE, 2013b).

\section{Fatores de Risco}

A doença apresenta etiologia multifatorial, como recém-nascidos de parto prematuro, suplementação de oxigênio prolongada, hiperglicemia, transfusão sanguínea, sepse, gestação múltipla, hemorragia interventricular, luminosidade excessiva, fototerapia, pré-disposição genética, baixo ganho ponderal, entre outros. E como fator de proteção à ROP grave, inclui-se mãe com pré-eclâmpsia, porém a fisiopatologia para tal ainda não é bem definida (ALAJBEGOVIC-HALIMIC J, 2015).

\section{Diagnóstico}

Como previamente estabelecido, o exame ocular, a fundoscopia, deve ser indicado a partir de 28 dias, idealmente entre a quarta e sexta semanas de vida para RNPT. Salienta-se que um oftalmologista eficiente é a base do diagnóstico oportuno da ROP. Quando oftalmologistas não estão presentes ativamente nos hospitais neonatais, neonatologistas ou pediatras devem ser capacitados a indicarem tal exame. Quanto a ida destes profissionais ao local para os exames específicos, é essencial a padronização de rotina, para ser identificada a criança que necessita. O aparelho utilizado pelo profissional é o oftalmoscópio indireto, o qual deve ser disponibilizado na Unidade (OPAS, 2018; CBO, 2017/2018). 
Obtém-se maior eficácia para realização da fundoscopia quando dilatação pupilar está máxima (efetiva midríase). É usado para tal, colírios de associações anticolinérgicos e agonistas do simpático, como por exemplo, tropicamida e fenilefrina, respectivamente. Pode-se repetir a instilação, se necessário, em intervalo de 5 minutos. Atentar-se para os efeitos colaterais locais ou sistêmicos causados por tais medicações, como conjuntivite, hiperemia conjuntival, aumento da pressão intraocular, reações psicóticas e distúrbios de comportamentos, os quais geralmente são transitórios. Se RN ainda apresentar-se agitado durante realização do exame, considerar instilação de colírio anestésico, contenção com ajuda da enfermagem e considerar oferta de mínima quantidade de glicose ao RN (MINISTRY OF HEALTH \& FAMILY WELFARE GOVERNMENT OF INDIA, 2017; CONSELHO FEDERAL DE MEDICINA (CFM), 2013). Alguns métodos complementares de exames têm sido utilizados, como mostra o Quadro 1.

Quadro 1 - Métodos complementares no diagnóstico Retinopatia da Prematuridade (ROP).

\begin{tabular}{|l|l|}
\hline \multicolumn{1}{|c|}{ TIPO DE EXAMES } & \multicolumn{1}{|c|}{ FUNDAMENTOS } \\
\hline Tomografia de coerência óptica & $\begin{array}{l}\text { Através da emissão de luz infravermelha permite avaliar cortes } \\
\text { seccionais das camadas de retina e coróide, e detectar se há algum } \\
\text { dano estrutural das mesmas. Avaliam também prognóstico de } \\
\text { recuperação visual após procedimentos. }\end{array}$ \\
\hline $\begin{array}{l}\text { Ecografia Doppler da Artéria } \\
\text { Oftálmica }\end{array}$ & $\begin{array}{l}\text { Estima a velocidade longitudinal do fluxo circulatório da artéria } \\
\text { oftálmica (artéria central da retina). E indicar necessidade } \\
\text { retratamento em ROP grave. }\end{array}$ \\
\hline Angiografia fluoresceínica & $\begin{array}{l}\text { Realizado através de fotografias constrastadas do fundo de olho após } \\
\text { administração de sistêmica de corantes de fluoresceína. Permite } \\
\text { avaliar a circulação retiniana e coroidiana, bem como áreas de } \\
\text { isquemia, extravasamentos vasculares e neovascularizações } \\
\text { retininias. }\end{array}$ \\
\hline Telemedicina & $\begin{array}{l}\text { Utiliza formas bidirecionais. Ensinam médicos e pacientes, } \\
\text { potencializando um maior cuidado aos olhos e realizam uma segunda } \\
\text { opinião diagnóstica de longe. Os meios mais comuns são através de } \\
\text { softwares, email e videoconferências, utilizando fotografias digitais } \\
\text { capturadas por retinógrafos portáteis especiais (RetCam). }\end{array}$ \\
\hline Eletrorretinografia & $\begin{array}{l}\text { Afere os potenciais evocados visuais a partir do tempo de transmissão } \\
\text { do sinal visual da retina para o córtex occipital. Além de investigar } \\
\text { mudanças na arquitetura celular da retina e vias centrais relevantes, } \\
\text { incluindo córtex visual primário e áreas corticais visuais extra-estriada. }\end{array}$ \\
\hline
\end{tabular}

Fonte: Araújo TN, et al., 2021; dados extraídos de Spandau U e Kim SJ, 2019; Khanamiri HN, et al., 2017; CBO, 2017-2018.

A fim de investigar mudança da arquitetura celular da retina e vias centrais relevantes, incluindo o córtex visual primário e áreas corticais visuais extra-estriadas, um importante exame vem ganhando espaço, a eletrorretinografia, onde os potenciais evocados visuais aferem o tempo de transmissão do sinal visual da retina para o córtex occipital (MINISTRY OF HEALTH \& FAMILY WELFARE GOVERNMENT OF INDIA, 2017).

\section{Diagnósticos diferenciais}

Dentre os principais diagnósticos diferenciais de ROP, encontra-se: Doença de Coats, Síndrome de Norrie, Toxocoríase Ocular, Vitreorretinopatia Exsudativa Familiar.

Doença de Coats, apresentar mal formação dos vasos retinianos desencadeando a passagem e o acúmulo de lipoproteínas para o local, favorecendo o rompimento da membrana limitante externa resultando em descolamento exsudativo; a Síndrome de Norrie, caracterizada por displasia retiniana hereditária e se manifesta com duplo deslocamento congénito da retina de caráter (SPANDAU U e KIM SJ, 2019); a 
Toxocoríase Ocular, é causada por um ascarididae de cão e gato, o Toxocara canis e Toxocara cati, onde suas larvas levam a inflamação ocular com formação de granulomas no polo posterior, acometendo após os seis meses de idade; e a Vitreorretinopatia Exsudativa Familiar, é uma doença autossômica dominante (cromossomo: 11q) de traço recessivo ligado ao X, os homens afetados têm filhos normais e filhas portadoras, e esta poderá ter filhos com problemas (CBO, 2017-2018).

\section{Classificação}

A Classificação Internacional de ROP (ICROP) original, descrito primeiro em uma publicação de 1984 e expandido em 1987, forneceu uma base sobre a qual vários ensaios de tratamento e estudos observacionais realizados. Nesse período, foi descrito sobre a localização do envolvimento da retina por zona; da extensão do envolvimento da retina por hora; do estágio ou gravidade da retinopatia na junção da retina vascularizada e avascular; e a presença ou ausência de vasos polares posteriores dilatados e tortuosos (mais doença).

Em 2005, foi realizada uma revisão e atualização da ICROP em um único documento que incluíram os seguintes: a) Conceito de uma retinopatia mais virulenta geralmente observada em bebês de menor peso ao nascer - ROP posterior agressiva (ROP-AP); b) Descrição de um nível intermediário de dilatação vascular e tortuosidade (doença pré-positiva) entre a vasculatura do pólo posterior de aparência normal e doença franca plus que apresenta dilatação e tortuosidade marcadas dos vasos do pólo posterior; e c) Esclarecimento da extensão da zona I (CBO, 2011).

\section{Estudos epidemiológicos}

De acordo com os fatores de risco apresentados para ROP e diagnósticos de ROP comparativos a países desenvolvidos e subdesenvolvidos, a literatura aponta estudos da correlação de oxigenoterapia e ROP.

Vários estudos demonstram que RNPT, com IG $<28$ semanas, e mantido em saturação de $\mathrm{O}_{2}$ entre 85$89 \%$ houve decréscimo da taxa de incidência ( $\mathrm{TI})$ de ROP, mas a taxa de mortalidade (TM) foi próxima de $16 \%$. Um estudo de coorte, ao compararem grupos de prematuros (IG $<32$ semanas, $P N \leq 1500 \mathrm{~g})$ que receberam $\mathrm{O}_{2}$ máximo $95 \%$ e mínimo $90 \%$, mostrou que o decréscimo do $\mathrm{O}_{2}$ não diminui significativamente a incidência da doença e que o PN e IG estavam significativamente associados ao surgimento da ROP, além de evidenciar um risco $6 x$ maior em meninos. Nessa coorte foram diagnosticados ROP em ambos os grupos sem valor significativo, porém a < IG influenciou no aparecimento da ROP $(p=0,002)$ e a correlação dos casos de ROP versus óbitos não obteve significância (OKAMOTO TC, et al., 2019; STENSON BJ, et al., 2013).

No que se refere a variável sexo, observa-se significância demonstrada para um ou outro, ou sem significância em ambas as variáveis. A exemplo recente em estudo quantitativo e transversal, não apresentou uma relação significativa para o desenvolvimento do ROP. Essa avaliação partiu da pesquisa documental de formulário (111 formulários de RN) de exame de retinopatia da prematuridade do grupo ROP Brasil, em crianças de IG 28-36 semanas e/ou PN $\leq 1500 \mathrm{~g}$ e com triagem oftalmológica (PAIVA AKL, et al., 2021).

Os estudos mostram que um nível de saturação de $\mathrm{O}_{2}$ mais baixo reduz significativamente a incidência grave de ROP, mas pode, ao mesmo tempo, aumentar a TM. O aumento da mortalidade é uma grande preocupação, uma vez que uma faixa-alvo mais baixa de saturação $\mathrm{O}_{2}$ está sendo cada vez mais defendida para prevenir a ROP (CBO, 2017-2018).

Estudo prospectivo realizado na China mostrou incidência em lactentes (RNPós-Termo e RNPT) uma proporção de ROP grave 1:5, respectivamente; associado a IG de 28 semanas apresentaram $55,8 \%$ mais risco do que os mais maduros $\leq 32(26,8 \%)$ semanas ao desenvolvimento da ROP (XU Y, et al., 2013).

\section{Acompanhamento e tratamento da ROP}

O acompanhamento da ROP, segundo o Quadro 2, depende do diagnóstico inicial e classificação. Aproximadamente $6 \%$ dos casos necessitam cirurgia corretiva. Nas retinopatias zona I e II, sem plus, a conduta médica é expectante. Se a ROP atingir Zona III, Estágios I e II, III (com menos de 5 cinco setores), sugere-se acompanhamento e mapeamento seriado da retina. Na forma grave da doença - pré-limiar tipo 1 ou AP - ROP está indicado tratamento por ablação sob analgesia e sedação ou anestesia geral (Quadro 2). 
Para o tratamento da ROP foram feitos vários ensaios clínicos com o objetivo de ablação da retina periférica avascular. Acreditava-se que reduziria a expressão de fatores angiogênicos promovendo assim a regressão da neovascularização pré-retiniana e prevenindo estágios avançados da doença, como descolamentos fibrovasculares. O "Multicenter Trial of Cryotherapy for ROP" demonstrou que a crioterapia reduziu o risco em pacientes que, segundo alguns critérios feitos por eles, tinham uma chance de $50 \%$ de um resultado indesejado. Mais tarde o estudo "Early Treatment for ROP" encontrou eficácia com o tratamento a laser em um estágio menos grave (ROP tipo 1) em que a chance de evolução para formas graves era de $15 \%$. Mais recentemente estudos vem demonstrando que injeções intravítreas de anti-VEGF (anti-fator de crescimento endotelial vascular) poderiam inibir a neovascularização patológica e facilitar a vascularização normal da retina previamente avascular (HARTNETT ME, 2020).

Quadro 2 - Acompanhamento de Retinopatia da Prematuridade (ROP).

\begin{tabular}{|l|l|}
\hline \multicolumn{1}{|c|}{ CARACTERÍSTICA DA RETINA } & \multicolumn{1}{c|}{ CUIDADOS E OBSERVAÇÕES } \\
\hline 1.Retina Madura (vascularização completa) & $\begin{array}{l}\text { Seguimento com 6 meses (avaliação de desenvolvimento } \\
\text { visual funcional, estrabismo, ametropias). Prematuros com } \\
46 \% \text { de chance para alterações oftalmológicas }\end{array}$ \\
\hline $\begin{array}{l}\text { 2.Retina Imadura (vascularização } \\
\text { incompleta ou presença de ROP < pre- } \\
\text { limiar) }\end{array}$ & Avaliação de 2/2 semanas \\
\hline 3.Retinopatia em regressão & Avaliação de 2/2 semanas \\
\hline 4.Retina Imatura, zona I & Exames semanais \\
\hline 5.ROP pré-limiar tipo 2 & Exames 3-7 dias \\
\hline $\begin{array}{l}\text { 6.ROP pré-limiar tipo 1 (zona I, qualquer } \\
\text { estágio com plus; zona I, estágio 3; zona II, } \\
\text { estágio 2 ou 3 plus) e limiar }\end{array}$ & Tratamento em até 72h \\
\hline 7.Os exames podem ser suspensos & $\begin{array}{l}\text { Vascularização da retina completa, IG de } 45 \text { semanas e } \\
\text { ausência de ROP pré-limiar, ROP completamente regredido. }\end{array}$ \\
\hline
\end{tabular}

Fonte: Araújo TN, et al., 2021; dados extraídos de Cervellini PM e Souza ABG, 2015.

Os resultados de um estudo retrospectivo realizado durante dois anos, em hospitais de referência na região amazônica, com 445 prematuros IG $\leq 30$ semanas e/ou $P N \leq 1500 \mathrm{~g}$, demonstrou que a maioria dos bebês desenvolveram ROP do tipo 1 e foram tratados com laser. Nesse tipo de estudo o estágio 0 , teve uma tendência significativa ( $p<0.05$ ) em 51,4\% RN (PAIVA AKL, et al., 2021; PIERMAROCCH S, et al., 2017).

\section{Prevenção e suplementação de oxigênio}

Existe um projeto no Brasil, criado pelo Instituto Fernandes Figueira (IFF), ligado à Fundação Fundação Oswaldo Cruz (FIOCRUZ), o projeto Controlando Oxigênio Alvo Ativo (COALA), e tem como objetivo exclusivamente otimizar o uso de oxigenoterapia suplementar durante hospitalização do RN prematuro. Esse projeto teve como base os estudos realizados em três países que demonstraram a saturação ideal de oxigênio para bebês prematuros extremos ( $\left.\mathrm{SO}_{2}: 91-95 \%\right)$ e isso pode evitar complicações No projeto COALA há 14 etapas, que se resumem desde a educação continuada da equipe multiprofissional ligada ao neonatal até a auditorias internas a cada mês para a boa conduta e conscientização diária (FIOCRUZ, 2019).

Uma abordagem multidisciplinar para a prevenção da cegueira por ROP, requer envolvimento de equipe multidisciplinar capacitada e treinada, composta por neonatologistas, enfermeiros, técnicos de enfermagem e oftalmologistas, no sentido de implementar programa eficaz de triagem e prevenção da cegueira pela ROP (MINISTÉRIO DA SAÚDE, 2014). 
É de suma importância ter na Unidade Neonatal, planejamento de programas de acompanhamento pré e pós-partos prematuros, a fim de adequar condições favoráveis para seu não aparecimento. Nesse âmbito, na impossibilidade de um oftalmologista na equipe, o (a) pediatra deve estar apto(a) a solicitar avaliação de fundo de olho. Também a equipe hospitalar deve garantir seguimento a longo prazo dos RNs de risco pós internação hospitalar, com consultas e acompanhamentos ambulatoriais, pré-agendados, o que configura medida essencial para garantir alta com seguridade dos RNs (OPAS, 2018; FONTES FILHO JB, et al., 2011).

Um outro desafio, é ampliar o conhecimento e incentivo de projetos de extensão e prevenção de Retinopatia em todos os hospitais neonatais do Brasil. Destaca-se também a importância de um pré-natal em gestantes de alto risco bem realizado a fim de evitar a prematuridade. Também de forma a incluir a decisão com precisão do momento mais oportuno para o uso de corticóide em gestantes antes de partos prematuros. Decisão esta, que pode ser em conjunto com pediatras, de forma a promover seguridade da mãe e filho(a) (CBO, 2017; CBO, 2018).

Em 2007, Fortes Filho e equipe chamavam atenção aos governos incentivarem mais políticas contra ROP e ampliar as informações sobre "Diretrizes de Atenção à Saúde Ocular na Infância", visto que também impacta diretamente no setor econômico e não só no SUS. Crianças com déficits visuais impactam aproximadamente em 70 anos de seguridade social, previamente nunca terem contribuído com verbas para tal finalidade. No Brasil, em 2018, cerca de 161 milhões de brasileiros que não possuem plano de saúde vem recebendo assistência oftalmológica, e um único objetivo: a busca pela melhoria das condições de saúde ocular da população (CBO, 2019).

Se a ROP é uma doença prevenível, logo o empenho da sociedade política governamental no mundo deve ser ímpar. A visão deve ser monitorada para a vida do novo ser e os pediatras ou neonatologiatas são as primeiras linhas de defesa da criança, devendo aplicar e fazer, além do necessário, para que a visão não seja tão prejudicada.

\section{CONSIDERAÇÕES FINAIS}

Dentre os instrumentos disponíveis para prevenção de ROP, torna-se imprescindível ter: uma equipe qualificada e treinada e protocolos de ROP instituídos em Unidades Neonatais, similar ao projeto Coala e diretrizes de atenção ocular na infância. Com base, nos fatores apresentados nesta Revisão Narrativa de Literatura, este procedimento de investigação de ROP deve ser reconhecido e potencializado individualmente, de forma que menos crianças venham manifestar deficiências secundárias a problemas visuais neonatais, tornando a criança menos prejudicial no desenvolvimento motor e capacidade de comunicação. Salienta-se que diagnóstico de ROP é também considerada um indicador de qualidade de cuidado integral ao RN.

\section{REFERENCIAS}

1. ALAJBEGOVIC-HALIMIC J. Risk Factors for Retinopathy of Prematurity in Premature Born Children. Med Arh, 2015; 69(6): 409-413.

2. AKKAWI MT, et al. Awareness of retinopathy of prematurity among pediatricians in West Bank, Palestine: a descriptive study. BMC Ophthalmol, 2018; 18(1): 195-199.

3. BLENCOWE $\mathrm{H}$, et al. Update on Blindness Due to Retinopathy of Prematurity Globally and in India. Indian Pediatr, 2016; 53(Supl 2): S89-92.

4. CARRION JZ, et al. Prevalencia of retinopathy of prematurity in Latin America. Clinical Ophthalmoly, 2011; 5: 16871695.

5. CERVELLINI PM, SOUZA ABG. Retinopatia da prematuridade. In: SOUZA ABG. Unidade de Terapia Intensiva Neonatal: Cuidados ao recém-nascido de médio e alto risco. São Paulo: Atheneu, 2015; 633 p.

6. CONSELHO BRASILEIRO DE OFTALMOLOGIA (CBO). As Condições de Saúde Ocular no Brasil. $2019 ; 104$ p. Disponível em: https://www.cbo.com.br/novo/publicacoes/condicoes_saude_ocular_brasil2019.pdf. Acesso em: 17 de setembro de 2021.

7. CONSELHO BRASILEIRO DE OFTALMOLOGIA (CBO). Sociedade Brasileira de Pediatria. Projeto Diretrizes. Retinopatia da prematuridade. São Paulo: Associação Médica Brasileira/Conselho Federal de Medicina, $2011 ; 16$ p. Disponível em: Projeto Diretrizes. Retinopatia da prematuridade. São Paulo: Associação Médica Brasileira/Conselho Federal de Medicina, 2011. Acesso em: 17 de setembro de 2021.

8. CONSELHO BRASILEIRO DE OFTALMOLOGIA (CBO). Oftalmologia Pediátrica e Estrabismo. Série Oftalmologia

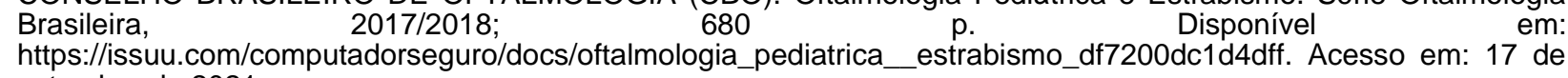
setembro de 2021. 
9. CONSELHO FEDERAL DE MEDICINA (CFM). Processo-Consulta CFM № 9/13 - Parecer CFM № 7/13. Disponível em: https://sistemas.cfm.org.br/normas/arquivos/pareceres/BR/2013/7_2013.pdf. Acesso em: 17 de setembro de 2021.

10. EJZENBAUM J, et al. Teste do reflexo vermelho. SBP Documento Científico, 2018; 1: 1-5.

11. FRANÇA-FREITAS MP, GIL MSCA. O Desenvolvimento de Crianças Cegas e de Crianças Videntes. Rev. Bras. Ed. Esp., Marília, 2012; 18(3): 507-526.

12. FONTES FO JB, et al. Prevention of retinopathy of prematurity. Arq Bras Oftalmol, 2011; 74(3): $217-221$.

13. FUNDAÇÃO OSWALDO CRUZ (FIOCRUZ). Instituto Nacional Fernandes Figueredo (INIFF). Controle do Oxigênio alvo para $\mathrm{O}$ uso seguro do $\mathrm{O}_{2}$ em unidades neonatais. 2019. Disponível em: https://portaldeboaspraticas.iff.fiocruz.br/wp-content/uploads/2019/01/CONTROLE_OXIGENIO_ALVO_COALA.pdf. Acesso em: 17 de setembro de 2021.

14. HARTNETT ME. Retinopatia da prematuridade: tratamento em evolução com fator de crescimento endotelial antivascular. Am J Ophthalmol, 2020; 218: 208-213.

15. KHANAMIRI HN, et al. Smartphone Fundus Photography. Medicine, 2017.

16. MIN HY, et al. Baixa visão: conhecendo mais para ajudar melhor. São Paulo: CBO/Laramara, 2018; 28p.

17. MINISTÉRIO DA SAÚDE. Secretaria de Atenção à Saúde. Atenção à saúde do recém-nascido: guia para os profissionais de saúde. 2. ed. Brasília: Brasil, 2014; 194 p. Disponível em: https://bvsms.saude.gov.br/bvs/publicacoes/atencao_saude_recem_nascido_v1.pdf. Acesso em: 17 de setembro de 2021.

18. MINISTÉRIO DA SAÚDE. Atenção humanizada ao recém-nascido de baixo peso: Método Canguru. 2. ed. Brasília: $\begin{array}{llll}\text { Brasil, } & 2013 a ; & \text { p. } & \text { Disponível }\end{array}$ https://bvsms.saude.gov.br/bvs/publicacoes/atencao_humanizada_recem_nascido_canguru.pdf. Acesso em: 17 de setembro de 2021.

19. MINISTÉRIO DA SAÚDE. Diretrizes de Atenção à Saúde Ocular na Infância: detecção e intervenção precoce para prevenção de deficiências visuais. Brasília: Brasil, 2013b; 40 p. Disponível em: https://bvsms.saude.gov.br/bvs/publicacoes/diretrizes_atencao_saude_ocular_infancia.pdf. Acesso em: 17 de setembro de 2021.

20. MINISTÉRIO DA SAÚDE. Secretaria de Atenção à Saúde. Atenção humanizada ao recém-nascido: Método Canguru. 3. ed. Brasília: Brasil, 2017; $342 \quad$ p. https://bvsms.saude.gov.br/bvs/publicacoes/atencao_humanizada_metodo_canguru_manual_3ed.pdf. Acesso em: 17 de setembro de 2021.

21. MINISTRY OF HEALTH \& FAMILY WELFARE GOVERNMENT OF INDIA. Guidelines for universal eye screening in newborns including retinopathy of prematurity: rashtriya bal swasthya karyakram 2017; 100 p. Disponível em: https://nhm.gov.in/images/pdf/programmes/RBSK/Resource_Documents/Revised_ROP_GuidelinesWeb_Optimized.pdf. Acesso em: 17 de setembro de 2021.

22. OKAMOTO CT, et al. Retinopatia da Prematuridade: análise de uma tentativa de redução de danos. Rev Bras Oftalmol, 2019; 78(2): 117-121.

23. ORGANIZACIÓN PANAMERICANA DE LA SALUD (OPAS). Guía de práctica clínica para el manejo de la retinopatía de la prematuridad. Washington, D.C.: OPS, 2018; 160p. Disponível em: https://iris.paho.org/bitstream/handle/10665.2/34948/9789275320020_spa.pdf?sequence=6\&isAllowed= Acesso em: 17 de setembro de 2021.

24. PAIVA AKL, et al. Perfil clínico de recém-nascidos em investigação para a retinopatia da prematuridade em uma maternidade na região Amazônica. Research, Society and Development, 2021; 10(3): 1-16.

25. PIERMAROCCHI S, et al. Predictive algorithms for early detection of retinopathy of prematurity. Acta ophthalmologica, 2017; 95(2): 158-164.

26. SANTOS MA. Avaliações das funções visuais de recém-nascidos prematuros nos primeiros seis meses de vida. Dissertação (Mestrado em Psicologia) - Instituto de Psicologia. Universidade de São Paulo, São Paulo, $2019 ; 80$ p.

27. SOUZA ABG. Unidade de Terapia Intensiva Neonatal: Cuidados com Recém-Nascidos de Médio e Alto Risco. São Paulo: Atheneu, 2015; 632p.

28. SOCIEDADE DE PEDIATRIA DE SÃO PAULO. Exame oftalmológico em crianças: quando e por quê?


https://www.spsp.org.br/site/asp/recomendacoes/Rec81_Oftalmo.pdf. Acesso em: 17 de setembro de 2021.

29. SPANDAU U, KIM SJ. Pediatric Retinal Vascular Diseases: From Angiography to Vitrectomy. Switzerland: Springer, 2019. 257p.

30. STENSON BJ, et al. Oxygen saturation and outcomes in preterm infants. N Engl J Med, 2013; 368(22): $2094-2104$.

31. TARTARELLA MB, FONTES FO JB. Retinopatia da prematuridade. e-Oftalmo.CBO: Rev Dig Oftalmol. 2016; 2(4): 116.

32. WEBER J, KELLEY J. Nursing assessment of physical systems. Philadelphia: Wolters Kluwer Health/Lippincott Williams \& Wilkins, 2014;16:228-262p.

33. WORLD HEALTH ORGANIZATION (WHO). Neonatal Mortality Rate. 2019. Disponível em: https://data. worldbank.org/indicator/SH.DYN.NMRT?end=2019\&locations=BR\&start=1960 Acesso em: 23 de setembro de 2021.

34. XU Y, et al. Screening for Retinopathy of Prematurity in China: A Neonatal Units-Based Prospective Study, Shanghai - China. Invest Ophthalmol Vis Sci., 2013; 54(13): 8229-8236.

35. ZIN A, GOLE GA. Retinopathy of Prematurity Incidence Today. Clin Perinatol, 2013; 40(2): 185-200. 